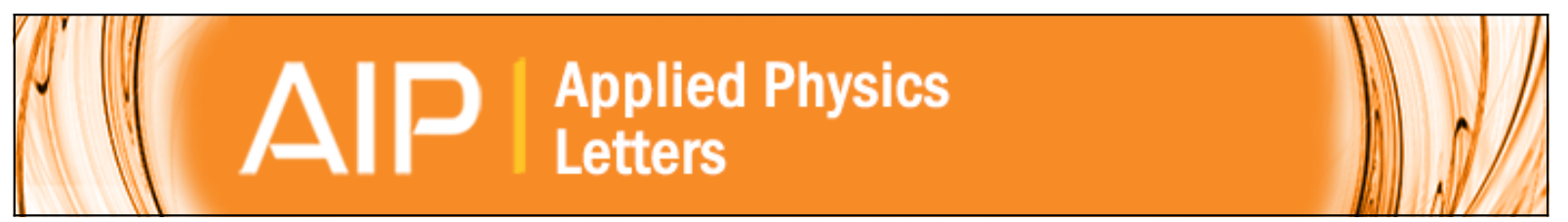

\title{
Stability of quantum-dot excited-state laser emission under simultaneous ground-state
} perturbation

Y. Kaptan, A. Röhm, B. Herzog, B. Lingnau, H. Schmeckebier, D. Arsenijević, V. Mikhelashvili, O. Schöps, M. Kolarczik, G. Eisenstein, D. Bimberg, U. Woggon, N. Owschimikow, and K. Lüdge

Citation: Applied Physics Letters 105, 191105 (2014); doi: 10.1063/1.4901051

View online: http://dx.doi.org/10.1063/1.4901051

View Table of Contents: http://scitation.aip.org/content/aip/journal/apl/105/19?ver=pdfcov

Published by the AIP Publishing

\section{Articles you may be interested in}

Comparison of dynamic properties of ground- and excited-state emission in p-doped InAs/GaAs quantum-dot lasers

Appl. Phys. Lett. 104, 181101 (2014); 10.1063/1.4875238

Ground-state power quenching in two-state lasing quantum dot lasers

J. Appl. Phys. 111, 043108 (2012); 10.1063/1.3682574

Effect of optical feedback to the ground and excited state emission of a passively mode locked quantum dot laser Appl. Phys. Lett. 97, 061114 (2010); 10.1063/1.3477955

Stable mode locking via ground- or excited-state transitions in a two-section quantum-dot laser

Appl. Phys. Lett. 89, 081124 (2006); 10.1063/1.2338767

Simultaneous two-state lasing in quantum-dot lasers

Appl. Phys. Lett. 82, 1818 (2003); 10.1063/1.1563742

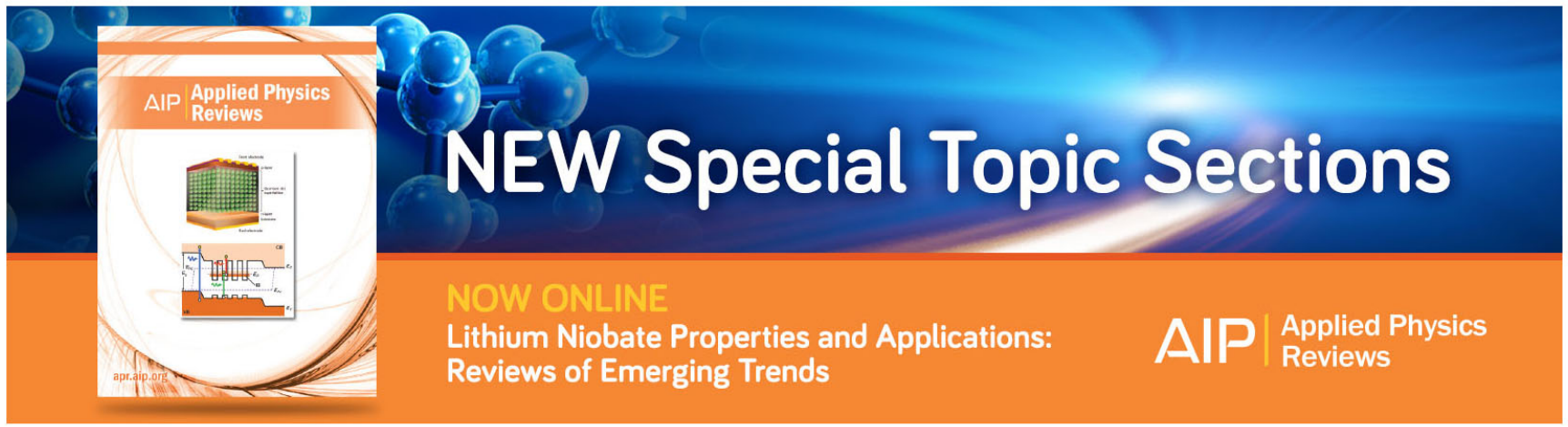




\title{
Stability of quantum-dot excited-state laser emission under simultaneous ground-state perturbation
}

\author{
Y. Kaptan, ${ }^{1, a)}$ A. Röhm, ${ }^{2}$ B. Herzog, ${ }^{1}$ B. Lingnau, ${ }^{2}$ H. Schmeckebier, ${ }^{3}$ D. Arsenijević, ${ }^{3}$ \\ V. Mikhelashvili, ${ }^{4}$ O. Schöps, ${ }^{1}$ M. Kolarczik, ${ }^{1}$ G. Eisenstein, ${ }^{4}$ D. Bimberg, ${ }^{3}$ U. Woggon, ${ }^{1}$ \\ N. Owschimikow, ${ }^{1}$ and K. Lüdge ${ }^{2}$ \\ ${ }^{1}$ Institut für Optik und Atomare Physik, Technische Universität Berlin, Berlin, Germany \\ ${ }^{2}$ Institut für Theoretische Physik, Technische Universität Berlin, Berlin, Germany \\ ${ }^{3}$ Institut für Festkörperphysik, Technische Universität Berlin, Berlin, Germany \\ ${ }^{4}$ Technion Institute of Technology, Faculty of Electrical Engineering, Haifa, Israel
}

(Received 12 June 2014; accepted 23 October 2014; published online 10 November 2014)

The impact of ground state amplification on the laser emission of $\operatorname{In}(\mathrm{Ga})$ As quantum dot excited state lasers is studied in time-resolved experiments. We find that a depopulation of the quantum dot ground state is followed by a drop in excited state lasing intensity. The magnitude of the drop is strongly dependent on the wavelength of the depletion pulse and the applied injection current. Numerical simulations based on laser rate equations reproduce the experimental results and explain the wavelength dependence by the different dynamics in lasing and non-lasing sub-ensembles within the inhomogeneously broadened quantum dots. At high injection levels, the observed response even upon perturbation of the lasing sub-ensemble is small and followed by a fast recovery, thus supporting the capacity of fast modulation in dual-state devices. (C) 2014 AIP Publishing LLC.

[http://dx.doi.org/10.1063/1.4901051]

Optoelectronic devices, like directly modulated or mode-locked lasers, based on semiconductor quantum dots (QDs) show superior performance compared to devices based on higher dimensional active layers. ${ }^{1}$ The devices display low threshold currents and an ultrafast gain recovery. ${ }^{2,3}$ Under operating conditions, the carriers being electrically injected into a two-dimensional (2D) reservoir accumulate at the band edge of this reservoir and are captured by the QDs on timescales of typically a few ps. ${ }^{4,5}$ At high injection levels, the capture rates are the limiting factor in the QD population recovery. ${ }^{6-9}$

Commonly, In(Ga)As-QDs exhibit more than one confined state, offering the possibility of dual- or multiple-state parallel operation, such as simultaneous lasing of the ground state (GS) and the first excited state (ES).$^{10}$ This so called two-state lasing has been well explored for steady-state conditions ${ }^{10-15}$ and can be explained by a pile-up of carriers in the ES due to slow ES-GS scattering. ${ }^{16,17}$ Until recently, alternative operation modes like dual-state amplification or mixed laser/amplifier operation, e.g., in an ES laser and GS optical amplifier, have not been investigated. The characterization of cross-interaction and dynamic influence between both emission channels is of crucial importance for the technical realization of dual-state quantum dot devices, which are designed to amplify modulated signals or modulate the emission of one state via an externally modulated input in the other state. In a recent publication, it was experimentally shown that the carrier recovery of the GS in a QD ES laser remains unaffected by the onset of lasing in the ES, which is promising for dual-state operation schemes in QD based optoelectronic devices. ${ }^{18}$

In this contribution, we focus on the complementary issue of stability of QD ES laser emission in presence of a

${ }^{a)}$ Electronic mail: yuecel.kaptan@physik.tu-berlin.de perturbation in the QD GS, which affects GS-ES transitions as well as the reservoir population and thus the reservoir-ES scattering channel. In time-resolved luminescence experiments and numerical simulations, we investigate the response of the ES laser emission to a signal pulse amplified at GS energies as a function of the injection current $J$ and the wavelength of the pulse. While $J$ controls the carrier population within the waveguiding region of the device (see the inset in Fig. 1), tuning the wavelength of the perturbing pulse within the GS shifts the perturbation between QD subensembles more or less resonant with the ES laser line.

The active zone of the investigated device consists of 5 stacks of undoped InGaAs/GaAs quantum dots grown by molecular beam epitaxy. Each dot layer is embedded in an InGaAs quantum well (DWELL structure). A shallow etched $1.33 \mathrm{~mm}$ long and $6 \mu \mathrm{m}$ wide ridge waveguide is used. The front facet (GS perturbation input) is as cleaved, whereas the

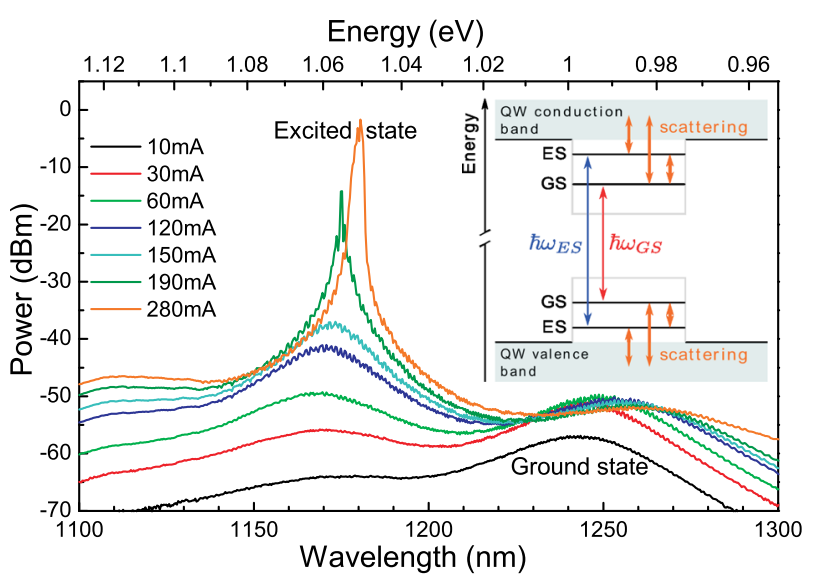

FIG. 1. Emission spectra of the QD device for a range of $J$. GS and ES emissions are clearly separated, and the onset of ES lasing is at about $190 \mathrm{~mA}$. Inset: Energy level scheme of the simulated QD-reservoir system. 
rear facet (ES laser output) has a dichroic mirror coating. The coating is composed of 18 alternating layers of $\mathrm{SiO}_{2}$ and $\mathrm{Ta}_{2} \mathrm{O}_{5}$ and has a reflectivity of $<5 \%$ for GS emission, suppressing the GS lasing for all operating currents. Below an injection current of $J=190 \mathrm{~mA}$, the device operates as an optical amplifier for GS and ES. Above this threshold, ES lasing occurs.

The electroluminescence spectra of the investigated QD ES laser are displayed in Fig. 1. For thermal stabilization, the device is mounted on a copper block with the excess heat being removed by water cooling. The emission from the QD GS is centered around $1250 \mathrm{~nm}(0.992 \mathrm{eV})$. It is broadened due to the inhomogeneous QD size and shape distribution with a full width at half maximum (FWHM) of $25 \mathrm{~nm}$ $(20 \mathrm{meV})$. At higher energies, amplified spontaneous emission (ASE) from the QD ES is observed $(1170 \mathrm{~nm} / 1.06 \mathrm{eV})$. Above a threshold current $J_{t h}^{E S}$ of $190 \mathrm{~mA}$, a narrow laser line emerges, also see Fig. 1.

Similar to the approach for time-resolving the ASE of a QD amplifier described in Ref. 9, we use a streak camera to detect the ES response to the GS perturbation. The experiment is illustrated schematically in Fig. 2. Laser pulses tunable through the QD GS energy were generated by an optical parametric oscillator from the fundamental pulses of a Ti:Sapphire oscillator (Coherent MIRA HP). The perturbing pulses have a temporal and spectral FWHM of $180 \mathrm{fs}$ and $17 \mathrm{~nm}$, respectively, at a repetition rate of $75.4 \mathrm{MHz}$. The luminescence from the device was imaged on the entrance slit of an Acton SPC2500 imaging spectrometer. The spectral coverage was about $55 \mathrm{~nm}$, allowing one to record the full width of the ES emission, while suppressing the strong background from the depletion pulse at GS energies. The spectrally dispersed signal from the device was imaged onto the IR sensitive S1 cathode of a Hamamatsu streak camera. The cathode slit setting of $20-40 \mu \mathrm{m}$ allowed for a temporal resolution of $30 \mathrm{ps}$, while recording a total time span of $2.2 \mathrm{~ns}$. The data acquisition was performed with a synchroscan streak unit in the analog integration mode for the readout CCD camera. The temporal dispersion in the streak camera is achieved by sweeping the optical signal across the detector area twice during one laser period, effectively folding the time-dependent signal back onto itself. In our case, the time-dependent signal already decays during the forward sweep, and the effect of the backsweep reduces to a constant offset. We account for this by

(a)
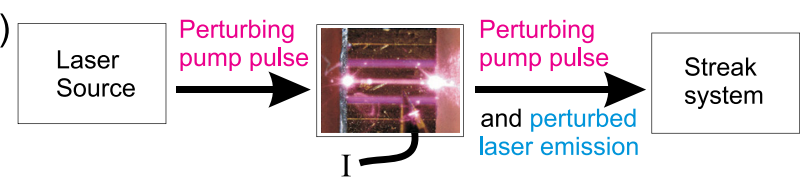

(b)
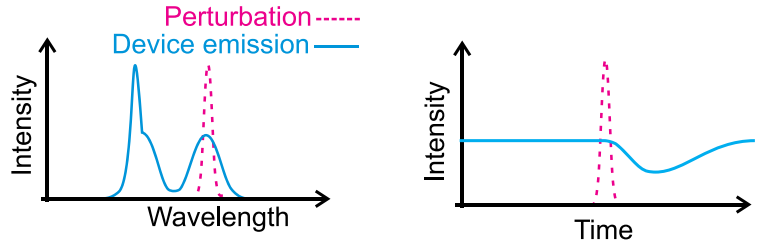

FIG. 2. (a) Schematic of the time-resolved experiment. (b) The emission from the device is spectrally dispersed in a monochromator, and the response of the ES emission to the perturbation pulse at GS energy is recorded. recording a reference trace without perturbation, dividing by a factor of 2 , and subtracting it from our data.

In order to capture all effects of the dual state device described above, we model the QD laser with a microscopically based multi-rate equation approach that has already proved to be able to quantitatively model the carrier dynamics within the waveguiding region as well as the dynamics of the emitted photons. ${ }^{719-21}$ We distinguish between the electrically pumped charge carrier reservoir with a $2 \mathrm{D}$ carrier density $w_{b}(b \in\{e, h\}$ denoting electrons and holes), and the localized QD ground state $(m=G S)$ and optically active first excited state $(m=E S)$ with their occupation probabilities given by $\varrho_{b, x}^{m}$. The energy levels are illustrated schematically in the inset of Fig. 1. In order to account for the inhomogeneous broadening of the QD ensemble, we distribute the QDs into a fraction $f^{a c t}$ of optically active QDs (resonant to the ES lasing wavelength, denoted by $x=a c t$ ) while the remaining $f^{\text {inact }}=\left(1-f^{a c t}\right)$ are denoted by $x=$ inact. The numerical value of $f^{a c t}$ is given by the overlap of homogeneous line shape and inhomogeneous QD distribution corrected for effects of spectral hole burning. ${ }^{22}$ In Ref. 19, it was shown that the best agreement with results obtained using the full model is achieved for $f^{a c t}=0.5$ at room temperature. The charge carrier dynamics are then given by

$$
\begin{gathered}
\frac{\mathrm{d}}{\mathrm{d} t} w_{b}=J-R_{l o s s}^{w} w_{e} w_{h}-2 N^{Q D} \sum_{\substack{x \in\{a c t, \text { inact }\} \\
m \in\{G S, E S\}}} \nu_{m} f^{x} S_{b, m, x}^{c a p}, \\
\frac{\mathrm{d}}{\mathrm{d} t} \varrho_{b, x}^{G S}=-W_{G S} \varrho_{e, x}^{G S} \varrho_{h, x}^{G S}+S_{b, G S, x}^{c a p}+S_{b, x}^{r e l}+\left.\frac{\partial \varrho_{b, x}^{G S}}{\partial t}\right|_{s t}, \\
\frac{\mathrm{d}}{\mathrm{d} t} \varrho_{b, x}^{E S}=-W_{E S} \varrho_{e, x}^{E S} \varrho_{h, x}^{E S}+S_{b, E S, x}^{c a p}-\frac{S_{b, x}^{r e l}}{2}+\left.\frac{\partial \varrho_{b, x}^{E S}}{\partial t}\right|_{s t} .
\end{gathered}
$$

Here, the pump current density per QW layer is denoted by $J, R_{\text {loss }}^{w}$ is an effective loss rate in the reservoir, $N^{Q D}$ is the 2D quantum dot density in each QW layer, $\nu_{m}$ is the degeneracy excluding spin of the $m$-th bound QD state, and $W_{m}$ is the spontaneous recombination rate in the QDs. The scattering terms describing the charge carrier capture and escape between reservoir and QD states, and the intra-dot relaxation are given by the following equations (see also the inset of Fig. 1):

$$
\begin{gathered}
S_{b, m, x}^{c a p}=S_{b, m}^{c a a, \text { in }}\left(1-\varrho_{b, x}^{m}\right)-S_{b, m}^{c a p, \text { out }} \varrho_{b, x}^{m}, \\
S_{b, x}^{r e l}=S_{b}^{r e l, \text { in }}\left(1-\varrho_{b, x}^{G S}\right) \varrho_{b, x}^{E S}-S_{b}^{r e l, \text { out }} \varrho_{b, x}^{G S}\left(1-\varrho_{b, x}^{E S}\right) .
\end{gathered}
$$

The in-scattering rates are determined by microscopic calculations of Auger-scattering processes, ${ }^{7}$ with their corresponding out-scattering rates given by detailed balance conditions. ${ }^{20}$ Here, we employ simplified fits to the full microscopic scattering rates. ${ }^{19}$ The stimulated emission is modeled by the following equations for photon density per QD layer at the ES resonance wavelength $N_{p h}^{E S}$, and the contribution to the dynamic equation of the QD occupations,

$$
\begin{aligned}
\frac{\mathrm{d}}{\mathrm{d} t} N_{p h}^{E S}= & {\left[2 g_{E S}^{a c t}\left(\varrho_{e, a c t}^{E S}+\varrho_{h, a c t}^{E S}-1\right)-2 \kappa\right] N_{p h}^{E S} } \\
& +4 N^{Q D} \beta W_{E S} \varrho_{e, a c t}^{E S} \varrho_{h, a c t}^{E S},
\end{aligned}
$$


TABLE I. Fit parameters.

\begin{tabular}{lcc}
\hline \hline Symbol & Value & Meaning \\
\hline$g_{G S}^{\text {act }}$ & $0.05 \mathrm{ps}^{-1}$ & GS linear gain (resonant) \\
$g_{G S}^{\text {inact }}$ & $0.025 \mathrm{ps}^{-1}$ & GS linear gain (off res.) \\
$g_{E S}^{\text {act }}$ & $0.1 \mathrm{ps}^{-1}$ & ES linear gain (resonant) \\
$g_{E S}^{\text {inact }}$ & $0 \mathrm{ps}^{-1}$ & ES linear gain (off res.) \\
$\kappa$ & $0.068 \mathrm{ps}^{-1}$ & Optical losses \\
$\beta$ & $1 \times 10^{-2}$ & Spontaneous emission factor \\
$N^{Q D}$ & $5 \times 10^{10} \mathrm{~cm}^{-2}$ & QD density per layer \\
$W_{G S}$ & $4.4 \times 10^{-4} \mathrm{ps}^{-1}$ & GS spontaneous emission rate \\
$W_{E S}$ & $5.5 \times 10^{-4} \mathrm{ps}^{-1}$ & ES spontaneous emission rate \\
$R_{\text {loss }}^{w}$ & $0.04 \mathrm{~nm}^{2} \mathrm{ps}^{-1}$ & QW loss rate \\
\hline \hline
\end{tabular}

$$
\left.\frac{\partial}{\partial t} \varrho_{b, x}^{m}\right|_{s t}=-\frac{2 g_{m}^{x}}{2 N^{Q D} \nu_{m} f^{x}}\left(\varrho_{e, x}^{m}+\varrho_{h, x}^{m}-1\right) N_{p h}^{m} .
$$

The gain coefficient of the $m$-th transition is denoted by $g_{m}^{x}$. It is assumed that only the resonant ES subgroups contribute to ES gain, while the off-resonant GS subgroups also contribute in part to the GS gain, due to the broader spectral width of the injected GS pulse. The cavity loss rate is given by $\kappa$. The values of the fitting parameters used in the simulations are given in Table I. Besides the microscopically determined scattering rates, the linear gain coefficients $g_{m}^{x}$ and losses $\kappa_{m}$, as well as the carrier loss rate $R_{\text {loss }}^{w}$ have the strongest influence on the time-dependence of the intensity. These parameters were chosen to yield the best agreement with the experimental data. Note, however, that those values only affect the quantitative results and not the qualitative effects discussed later.

The GS photon density is modeled by a single Gaussian pulse with amplitude $A_{0}$ and width $T_{\mathrm{FWHM}}=180 \mathrm{fs}$,

$$
N_{p h}^{G S}(t)=A_{0} \exp \left[-4 \log (2)\left(t / T_{\mathrm{FWHM}}\right)^{2}\right]
$$

Figure 3 shows the time-dependent intensity of the ES laser emission after a GS depletion pulse in the experiment

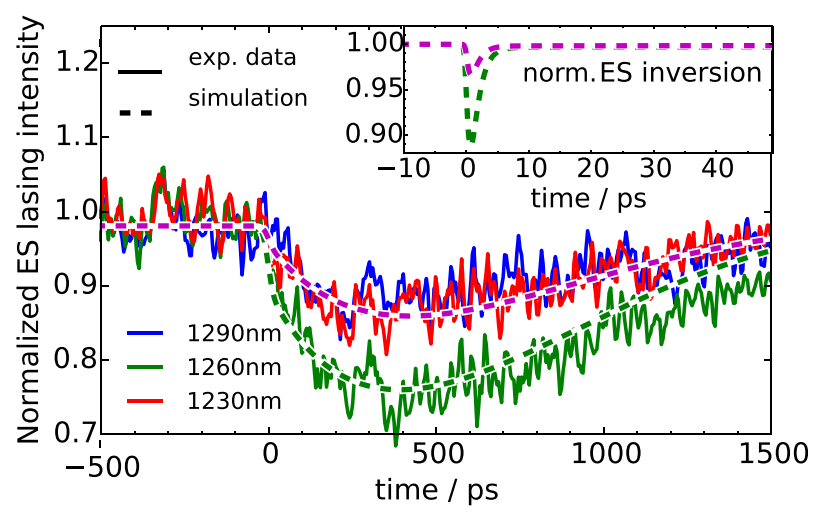

FIG. 3. Influence of GS pulse wavelength on the ES lasing dynamics for $J=200 \mathrm{~mA}$. Shown are the time evolution of the ES lasing intensity after an optical perturbation to the non-resonant QDs (centered around $1290 \mathrm{~nm}$ (blue line), $1230 \mathrm{~nm}$ (red line)) as well as to the resonant QDs at $1260 \mathrm{~nm}$ (green line). Dashed lines are the simulation results (off-resonant excitation is obtained by setting $g_{G S}^{a c t}=0.006 \mathrm{ps}^{-1}$ ). The inset shows the simulated ES population inversion dynamics immediately after the depletion pulse. (solid lines). The curves were corrected for the influence of the streak camera backsweep as discussed above. The ES laser was operated slightly above threshold, and the wavelength of the perturbing pulse depleting the GS was varied from $1230 \mathrm{~nm}$ to $1290 \mathrm{~nm}$, while keeping the averaged power constant at $3 \mathrm{~mW}$. In all cases, we observe a drop in the ES laser output intensity after the depletion pulse, with the steepest slope and maximal relative reduction for a wavelength of $1260 \mathrm{~nm}$. A similar spectral dependence of the response to a perturbation was observed by us also in Ref. 18 for the GS recovery in an ES laser. Considering that the laser line is narrow compared to the ASE background, cf. also Fig. 1, we assume that only a fraction $f^{a c t}$ of the QDs contributes to the laser emission, and that this active sub-ensemble is located energetically at the wavelength of $1260 \mathrm{~nm}$. This assumption is substantiated by our numerical simulations (dashed lines), which excellently reproduce the observed dynamics if we divide the QDs into active and non-active sub-ensembles, and locate the fully active sub-ensemble at $1260 \mathrm{~nm}$. The numerical results were convoluted with a Gaussian of $30 \mathrm{ps}$ width to account for the experimental time resolution. Note that the difference between the limiting cases is not only qualitative, but also quantitative. In case the ES of active QDs is depleted, the response is steep emission reduction at short times. This reflects the sudden reduction in ES population inversion by the depletion pulse, as is illustrated in the inset of Fig. 3. In case the direct scattering channel from ES to GS reduces the ES inversion below the level needed to facilitate lasing, this leads to the steep decay of ES emission (Fig. 3, green dashed line). In the case of non-active QDs, this channel is absent, hence the slower decay and shallower minimum for depletion pulses at $1230 \mathrm{~nm}$ and $1290 \mathrm{~nm}$. Interestingly, also in these cases, the fast dynamics can be only accounted for if a small fraction of active QDs is introduced (Fig. 3, violet dashed line). The slow recovery in both cases occurs with the same rate, as it is governed by the re-equilibration dynamics of the complete system.

The response of the laser emission to a perturbation in the GS of the lasing sub-ensemble is displayed in greater detail in Fig. 4(a). The injection current was tuned from below the laser threshold (black curve), through the threshold $\left(J_{t h}^{E S}=190 \mathrm{~mA}\right)$ (dark red curve), and above the threshold (bright red to yellow curves). The dashed curves show the numerical results which nicely reproduce the experimental data. In all cases, the ES emission reacts to the perturbation by forming a dip in its intensity. The time and relative depth of the ES emission change versus $J$ are plotted in Fig. 4(b). It prominently shows the nonlinear dependence on $J$ which is, within the experimental error, correctly described by our simulations. The drop in the ES emission intensity is sharp, followed by a recovery on a timescale of a few 100 ps, characteristic for the reservoir recovery at intermediate injection levels. ${ }^{5}$ While QD and QW carrier levels are in a quasi-equilibrium after a few scattering time scales, i.e., several picoseconds (cf. the inset of Fig. 3), the perturbation on the lasing system induces relaxation oscillations (ROs). The RO frequency $\omega_{R O}$ and damping $\Gamma_{R O}$ can be approximated by single- 


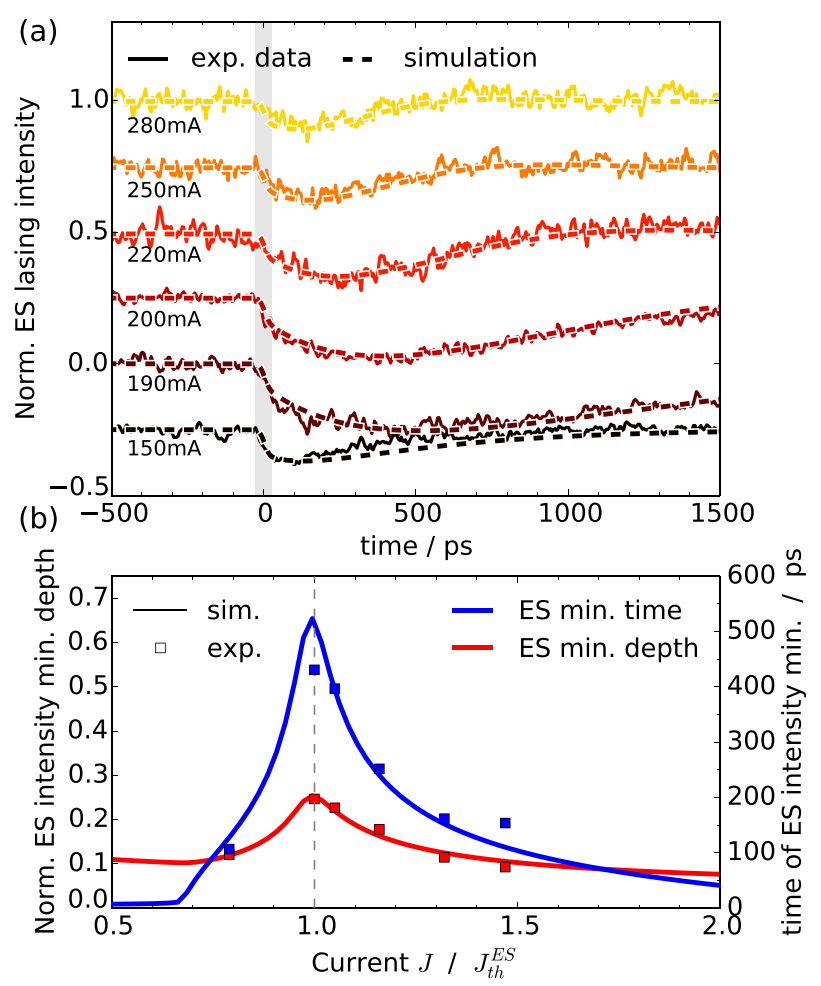

FIG. 4. Pump current dependence of ES lasing intensity. (a) Time evolution of ES laser intensity after a Gaussian shaped perturbation at GS wavelength $(\lambda=1260 \mathrm{~nm})$ at $($ time $=0)$. The curves have been shifted vertically by -0.25 for subsequent $J$ for better readability. Shown are experimental data (solid lines) and simulation results (dashed lines). (b) Relative depth (red) and position in time (blue) of the ES intensity minimum shown in (a) for numerical (lines) and experimental(squares) data in dependence of $J$ (normalized to the ES threshold $J_{t h}^{E S}=190 \mathrm{~mA}$ ).

state lasing behaviour and calculated by evaluating the eigenvalues of the stable lasing state, as is done in Sec. 1.2 of Refs. 21 and 23 ,

$$
\begin{gathered}
\omega_{R O} \propto \sqrt{J-J_{t h}}, \\
\Gamma_{R O} \propto J .
\end{gathered}
$$

Hence, the timing of the minimum is linked to the RO frequency, while the minimum depth can be explained as a combination of RO damping and the relative intensity loss $\Delta I / \Delta J$, which is highest at threshold. Below the laser threshold, we see the pure population depletion effect and spontaneous emission processes. At threshold, the dip in the ES emission is most pronounced and displays a slow onset and a slow recovery, which can be explained by the trans-critical bifurcation taking place at the laser threshold. The ROs become faster with increasing injection level while the damping increases (compare yellow line in Fig. 4(a)) with $J$, hence the time and depth of the ES intensity minimum are smaller for higher injection currents. ${ }^{21,24}$

In conclusion, we experimentally and numerically investigated the effect of GS depletion by an ultrashort laser pulse on the laser emission of a QD ES laser. The observed response is a dip in the laser emission, the depth and recovery rate of which depend on the wavelength of the GS depletion pulse and the injection current. At threshold, we see a critical slowing down of the ES laser reaction time. Resonant (lasing) QDs are most affected, with a temporary reduction of the laser emission by more than $20 \%$. The effect of the depletion pulse is greatly reduced for high injection current. The observed dynamics is quantitatively well described by our numerical model for all current injection levels below and above the laser threshold. It shows that increased reservoir filling at high injection current leads to a faster transition back to equilibrium through fast scattering processes and reservoir-induced damping. A time-varying perturbation in the QD GS, as would be caused by an optical telecommunications signal, could thus be processed simultaneously in a device lasing in the QD ES, provided the current injection level was high.

We are grateful to Nicolai B. Grosse for his critical analysis of the manuscript. This work was supported by Sfb 787 from the German research foundation and the Alexander von Humboldt foundation. M.K. acknowledges support from German research foundation via GRK 1558.

${ }^{1}$ P. Bhattacharya, S. Ghosh, and A. D. Stiff-Roberts, Annu. Rev. Mater. Res. 34, 1 (2004).

${ }^{2}$ P. Borri, W. Langbein, J. M. Hvam, F. Heinrichsdorff, M.-H. Mao, and D. Bimberg, IEEE Photon. Technol. Lett. 12, 594 (2000).

${ }^{3}$ T. W. Berg, S. Bischoff, I. Magnusdottir, and J. Mørk, IEEE Photon. Technol. Lett. 13, 541 (2001).

${ }^{4}$ T. Piwonski, I. O'Driscoll, J. Houlihan, G. Huyet, R. J. Manning, and A. V. Uskov, Appl. Phys. Lett. 90, 122108 (2007).

${ }^{5}$ J. Gomis-Bresco, S. Dommers, V. V. Temnov, U. Woggon, J. MartinezPastor, M. Laemmlin, and D. Bimberg, IEEE J. Quantum Electron 45, 1121 (2009).

${ }^{6}$ D. R. Matthews, H. D. Summers, P. M. Smowton, and M. Hopkinson, Appl. Phys. Lett. 81, 4904 (2002).

${ }^{7}$ N. Majer, K. Lüdge, and E. Schöll, Phys. Rev. B 82, 235301 (2010).

${ }^{8}$ J. Gomis-Bresco, S. Dommers, V. V. Temnov, U. Woggon, M. Laemmlin, D. Bimberg, E. Malic, M. Richter, E. Schöll, and A. Knorr, Phys. Rev. Lett. 101, 256803 (2008).

${ }^{9}$ J. Gomis-Bresco, S. Dommers-Völkel, O. Schöps, Y. Kaptan, O. Dyatlova, D. Bimberg, and U. Woggon, Appl. Phys. Lett. 97, 251106 (2010).

${ }^{10}$ A. Markus, J. X. Chen, C. Paranthoën, A. Fiore, C. Platz, and O. GauthierLafaye, Appl. Phys. Lett. 82, 1818 (2003).

${ }^{11}$ Q. Cao, S. F. Yoon, C. Z. Tong, C. Y. Ngo, C. Y. Liu, R. Wang, and H. X. Zhao, Appl. Phys. Lett. 95, 191101 (2009).

${ }^{12}$ M. A. Cataluna, D. I. Nikitichev, S. Mikroulis, H. Simos, C. Simos, C. Mesaritakis, D. Syvridis, I. Krestnikov, D. Livshits, and E. U. Rafailov, Opt. Express 18, 12832 (2010).

${ }^{13}$ M. Gioannini, J. Appl. Phys. 111, 043108 (2012).

${ }^{14}$ E. A. Viktorov, P. Mandel, Y. Tanguy, J. Houlihan, and G. Huyet, Appl. Phys. Lett. 87, 053113 (2005).

${ }^{15}$ K. Veselinov, F. Grillot, C. Cornet, J. Even, A. Bekiarski, M. Gioannini, and S. Loualiche, IEEE J. Quantum Electron 43, 810 (2007).

${ }^{16}$ A. Markus, J. X. Chen, O. Gauthier-Lafaye, J.-G. Provost, D. Paranthoën, and A. Fiore, IEEE J. Sel. Top. Quantum Electron 9, 1308 (2003).

${ }^{17}$ V. V. Korenev, A. V. Savelyev, A. E. Zhukov, A. V. Omelchenko, and M. V. Maximov, Semiconductors 47, 1397 (2013).

${ }^{18}$ Y. Kaptan, H. Schmeckebier, B. Herzog, D. Arsenijevic, M. Kolarczik, V. Mikhelashvili, N. Owschimikow, G. Eisenstein, D. Bimberg, and U. Woggon, Appl. Phys. Lett. 104, 261108 (2014).

${ }^{19}$ B. Lingnau, W. W. Chow, and K. Lüdge, Opt. Express 22, 4867 (2014).

${ }^{20} \mathrm{~K}$. Lüdge, "Modeling quantum dot based laser devices," in Nonlinear Laser Dynamics - From Quantum Dots to Cryptography (WILEY-VCH, Weinheim, 2012), pp. 3-34.

${ }^{21}$ K. Lüdge, E. Schöll, E. Viktorov, and T. Erneux, J. Appl. Phys. 109, 103112 (2011).

${ }^{22}$ B. Lingnau, W. W. Chow, E. Schöll, and K. Lüdge, New J. Phys. 15, 093031 (2013).

${ }^{23}$ T. Erneux and P. Glorieux, Laser Dynamics (Cambridge University Press, UK, 2010).

${ }^{24} \mathrm{C}$. Otto, "Dynamics of quantum dot lasers-Effects of optical feedback and external optical injection," in Springer Theses (Springer, Heidelberg, 2014). 